\title{
ARrió Populaçãa em situação de rua no Brasil: estudo descritivo sobre o perfil sociodemográfico e da morbidade por tuberculose, 2014-2019
}

doi: $10.1590 / 51679-49742021000100029$

\author{
Street people in Brazil: a descriptive study of their sociodemographic profile and \\ tuberculosis morbidity, 2014-2019
}

Población sin hogar en Brasil: estudio descriptivo sobre el perfil sociodemográfico y la morbilidad por tuberculosis, 2014-2019

\author{
Tarcisio Oliveira Silva ${ }^{1}$ - (1) orcid.org/0000-0001-9937-9150 \\ Paulo Jorge de Souza Vianna' - (1) orcid.org/0000-0002-0181-6648 \\ Márlon Vinícius Gama Almeida² - (1) orcid.org/0000-0001-8026-1136 \\ Sélton Diniz dos Santos ${ }^{3}$ - (1) orcid.org/0000-0002-3992-4353 \\ Joilda Silva Nery' ${ }^{1}$ - (1) orcid.org/0000-0002-1576-6418 \\ 'Universidade Federal da Bahia, Instituto de Saúde Coletiva, Salvador, BA, Brasil \\ ${ }^{2}$ Universidade Federal do Vale do São Francisco, Colegiado de Medicina, Paulo Afonso, BA, Brasil \\ ${ }^{3}$ Universidade Estadual de Feira de Santana, Departamento de Saúde, Feira de Santana, BA, Brasil
}

\section{Resumo}

Objetivo: Caracterizar o perfil sociodemográfico da população em situação de rua (PSR) inscrita no Cadastro Único para Programas Sociais (CadÚnico) até 2019, e da morbidade por tuberculose nessa população notificada no Sistema de Informação de Agravos de Notificação (Sinan) no período 2014-2018. Métodos: Estudo descritivo sobre dados do Sinan e do Cadúnico. Resultados: Foram incluídas 127.536 pessoas cadastradas no Cadúnico e 14.059 com tuberculose notificadas no Sinan. Foram observadas maiores concentrações da PSR nos estados das regiões Sul e Sudeste. Nas duas subpopulações analisadas, predominou uma PSR do sexo masculino (CadÚnico = 86,8\%; Sinan $=80,9 \%$ ), de raça/cor da pele negra (CadÚnico = 67,5\%; Sinan =64,1\%) e escolaridade até o ensino médio incompleto (Cadúnico = 81,9\%; Sinan = 60,1\%). A alta proporção de abandono (39,0\%) do tratamento refletiu-se nos elevados percentuais de reingresso e recidiva. Conclusão: A tuberculose permanece como grave problema de Saúde Pública, que agrava a condição de sobrevivência nas ruas do Brasil.

Palavras-chave: Pessoas em Situação de Rua; Vulnerabilidade Social; Epidemiologia Descritiva; Tuberculose.

\section{Endereço para correspondência:}

Tarcisio Oliveira Silva - Rua Rodolpho Coelho Cavalcante, no 162, Ed. Morada das Torres, apt. 101, A. Jardim Armação, Salvador, BA, Brasil. CEP: 41750-166

E-mail: tosbahia@gmail.com 


\section{Introdução}

A população em situação de rua (PSR) constitui-se de um grupo heterogêneo, em situação de pobreza extrema, vínculos familiares fragilizados ou rompidos e moradia não convencional de caráter permanente ou temporário. ${ }^{1}$

\section{As ruas oferecem muitos perigos a esta população, como violência, insegurança alimentar, indisponibilidade de água potável, privação de sono e afetividade, expondo-a a várias doenças, agravose situações de vulnerabilidade.}

As ruas oferecem muitos perigos a essa população, como violência, insegurança alimentar, indisponibilidade de água potável, privação de sono e de afetividade, expondo-a a várias doenças, agravos e situações de vulnerabilidade. A escassez de informações sobre a PSR é uma realidade no Brasil e, até o momento desta publicação, não está prevista sua inclusão no censo demográfico do Instituto Brasileiro de Geografia e Estatística. $^{2-4}$

Pesquisa realizada pelo Instituto de Pesquisa Econômica Aplicada, fundação pública vinculada ao Ministério da Economia do Brasil, com estimativas projetadas a partir de informações do Cadastro Único para Programas Sociais do Governo Federal (CadÚnico) e do Sistema Único de Assistência Social, permite presumir que em 2020 existam 221.869 PSR no Brasil, predominantemente na região Sudeste, mas em crescimento bastante significativo no Norte do país. ${ }^{5}$

Em 2009, com a publicação do Decreto Federal $\mathrm{n}^{0} 7.053$, em 23 de dezembro de 2009, foi instituída a Política Nacional para a População em Situação de Rua. ${ }^{6}$ Entretanto, não se alcançou a plena garantia de direitos e condições para uma existência digna e proteção social dessa população até os dias de hoje, segundo a militância política do Movimento Nacional da População em Situação de Rua. No campo da Saúde, uma vez admitidas iniquidades no acesso a seus serviços, decidiu-se pelo desmembramento de estratégias de ação, como o 'Plano operativo para implementação de ações em saúde da população em situação de rua
2012-2015', pela (hoje extinta) Secretaria de Gestão Estratégica e Participativa do Ministério da Saúde, e o 'Consultório na Rua', um projeto da Política Nacional de Atenção Básica, coordenada nacionalmente pela Secretaria de Atenção Primária à Saúde do Ministério. Apesar dessas iniciativas, a PSR ainda é acometida por violências, adoecimento mental, doenças crônicas e doenças infecciosas, entre estas a tuberculose. ${ }^{7-9}$

0 Brasil é um dos 22 países com alta endemicidade para tuberculose. A Organização Mundial da Saúde estima que cerca de um quarto da população mundial seja portadora do Mycobacterium tuberculosis e 5 a $10 \%$ dos infectados desenvolvam a tuberculose ao longo da vida. ${ }^{3,10}$

A ausência de moradia e as precárias condições de vida são fatores determinantes para a ocorrência da doença na PSR. Segundo estudo realizado na capital Salvador, $\mathrm{BA},{ }^{11}$ a tuberculose aparece como a terceira maior causa de adoecimento nesse grupo específico. No estado de São Paulo, estudo com base em dados de 2009 a 2013 estimou a magnitude da tuberculose, cujos percentuais de abandono do tratamento entre a PSR são particularmente altos, chegando a 57,3\%. Pessoas em situação de rua apresentaram uma incidência 10 a 85 vezes superior de infecções latentes por tuberculose e doença ativa, quando comparadas à população geral. ${ }^{12}$ Por esses motivos, o Programa Nacional de Controle da Tuberculose inclui a PSR como população prioritária. ${ }^{13}$

Este estudo, determinado a visibilizar direitos humanos negados e apresentar dados que favoreçam a tomada de decisão pela realização de ações previstas em políticas públicas intersetoriais, teve como objetivo caracterizar (i) o perfil sociodemográfico da PSR registrada no CadÚnico em maio de 2019 e (ii) a morbidade por tuberculose nessa população notificada no Sistema de Informação de Agravos de Notificação (Sinan) no período de 2014 a 2018.

\section{Métodos}

Estudo descritivo de toda a PSR registrada no Tabulador de Informações do CadÚnico (TABCAD CadÚnico V7), em maio de 2019, ${ }^{14}$ e da PSR acometida por tuberculose e notificada no Sinan (TABNET) no período de 2014 a 2018..$^{15}$

Para a definição da população do estudo, foram utilizados os dados registrados no TABCAD até 0 ano de 
2019, uma vez que esse tabulador não permite recorte temporal. Esses dados foram acessados no dia 2 de maio de 2019. ${ }^{14}$ Para a escolha da população no Sinan, procurou-se abranger o intervalo de maior notificação dos casos observado, cujo início corresponde a 2014, quando se inseriu no sistema a categoria 'População em Situação de Rua', pela variável 'Populações Especiais' - dados tabulados a partir do TABNET, no dia 20 de setembro de 2020. ${ }^{15}$

Consultadas no CadÚnico, foram incluídas as seguintes variáveis:

a) sexo (masculino; feminino);

b) faixa etária (em anos: 0 a 15; 16 a 24; 25 a 44; 45 a 54; 55 a 64; 65 e mais);

c) raça/cor da pele (branca; preta; amarela; parda; indígena; sem informação);

d) escolaridade (por grau de instrução: sem instrução; ensino fundamental incompleto; ensino fundamental completo; ensino médio incompleto; ensino médio completo; ensino superior incompleto ou mais; sem informação);

e) faixa de renda familiar per capita (extrema pobreza; pobreza; baixa renda; acima de 1/2 salário mínimo); e

f) ser beneficiário do Programa Bolsa Família (sim; não). ${ }^{14}$

Para a caracterização da faixa de renda familiar per capita, adotou-se o critério do Ministério da Cidadania: 'extrema pobreza' como renda mensal por pessoa de até $R \$ 89,00$; e 'pobreza', como renda mensal por pessoa de $R \$ 89,01$ até $R \$ 178,00{ }^{16}$

$\mathrm{Na}$ caracterização dos casos de tuberculose notificados no Sinan, optou-se pela descrição das variáveis sociodemográficas equivalentes às obtidas do CadÚnico, com exceção da variável 'faixa de renda familiar per capita', esta não registrada na ficha do Sinan. Dada a especificidade do TABNET, adotou-se para a variável 'faixa etária' as seguintes categorias de idade, em anos: 0 a 14; 15 a 24; 25 a 34; 35 a 44; 45 a 54; 55 a 64; 65 e mais; sem informação. ${ }^{15}$ Também foram descritas as variáveis 'tipo de entrada' (caso novo; reingresso; recidiva; transferência; diagnóstico pós-óbito; não sabe) e 'desfecho do tratamento' (cura; abandono; óbito; transferência; ignorado/em branco) dos casos de tuberculose em PSR no Brasil. ${ }^{15}$ $\mathrm{Na}$ análise dos desfechos da tuberculose na população em situação de rua, foram excluídas as variáveis
'TB-DR', 'mudança de esquema', 'falência' e 'abandono primário', por apresentarem resultados inferiores a 1,1\%. Também foram excluídos os óbitos por outras causas, haja vista a impossibilidade de análise de causas secundárias. No período da coleta e análise dos dados, os encerramentos dos casos de 2019 não estavam consolidados no Sinan e, por esse motivo, esses dados para este ano não foram incluídos no estudo. ${ }^{15}$

Foram elaborados mapas pelo programa 0Gis, para análise comparativa das Unidades da Federação (UFs) com maior número de pessoas em situação de rua cadastradas no Cadúnico e que, também, apresentavam as maiores proporções de casos confirmados de tuberculose na PSR. A análise da PSR registrada no CadÚnico e no Sinan partiu do número absoluto e da proporção (\%) das variáveis de interesse, apoiada no software Excel.

Não foi necessária submissão do projeto do estudo a um Comitê de Ética em Pesquisa, visto que os dados secundários utilizados estavam disponíveis para o público, mediante consulta aos sistemas de informações.

\section{Resultados}

Foram identificadas 127.536 PSR registradas no CadÚnico em maio de 2019. Conforme o mapa da distribuição da PSR por UF, representado pela Figura 1A, a maior parte dessa população concentrava-se em Minas Gerais, São Paulo, Rio de Janeiro, Paraná e Rio Grande do Sul, em números superiores a 5 mil pessoas. As UFs com menos de 500 pessoas cadastradas nessa situação encontravam-se na região Norte: Tocantins, Amapá, Rondônia e Acre.

$\mathrm{Na}$ análise do número de casos de tuberculose na PSR notificados no Sinan, foram identificadas 14.059 pessoas com tuberculose entre 2014 e 2018. De acordo com a Figura 1B, em São Paulo e nos estados da região Sul, mais de $4 \%$ dos casos de tuberculose ocorreram nessa população. Vale destacar que entre os dez estados (e o Distrito Federal) que apresentaram entre 1 mil e 5 mil pessoas registradas no CadÚnico, apenas Goiás, Minas Gerais, Rio de Janeiro e Espírito Santo mantiveram proporções de casos de tuberculose de 3 a 3,9\%.

Na Figura 2, é apresentado o somatório das pessoas notificadas no Sinan com o modo de entrada 'reingresso após abandono', 'recidiva', 'transferência', 'diagnóstico pós-óbito' e dos registros 'sem informação' de tipo de entrada: no total, foram notificadas 


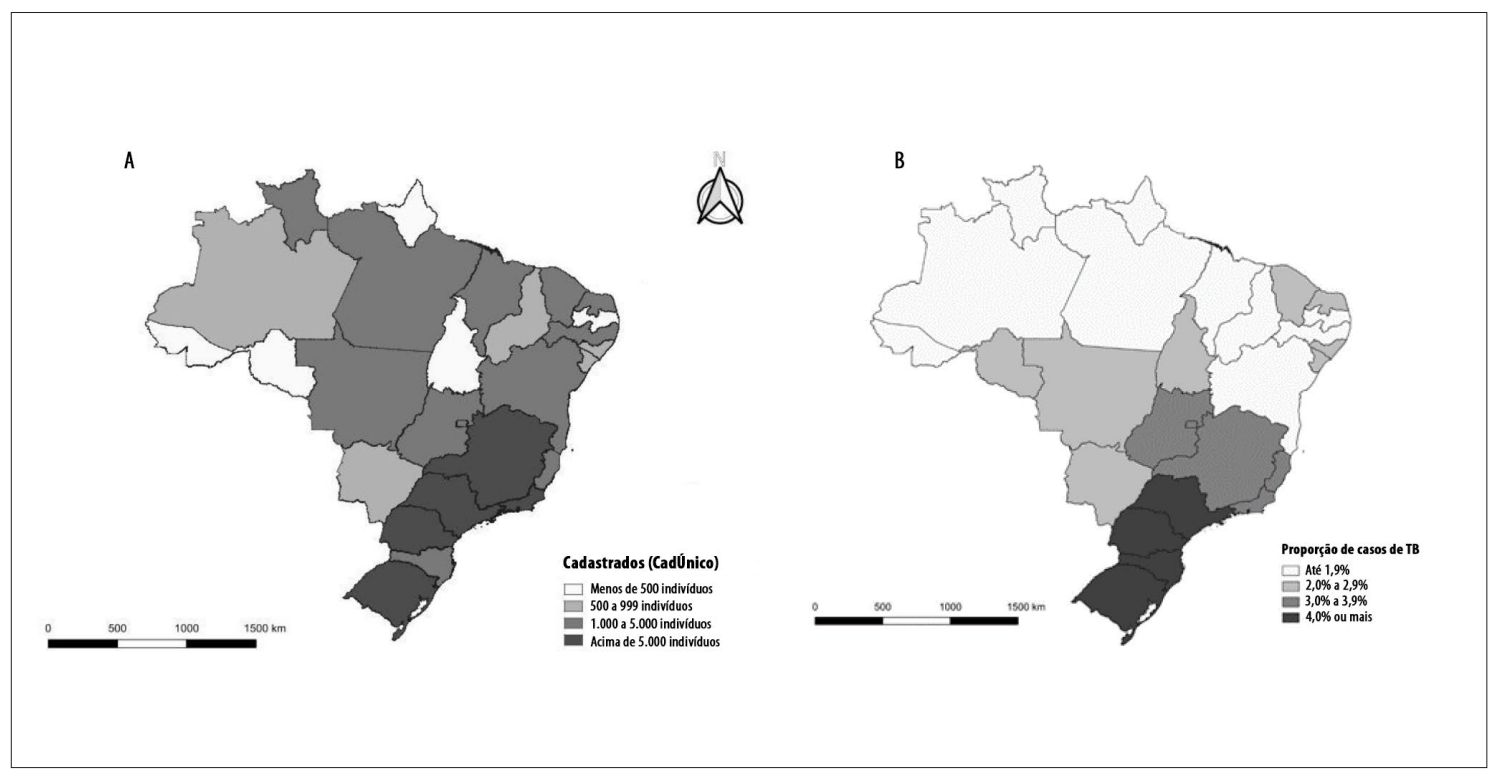

Figura 1 - Número de pessoas inscritas no Cadastro Único para Programas Sociais (CadÚnico) por Unidade da Federação (UF) em maio de 2019 (A) e proporção de casos confirmados de tuberculose (TB) entre pessoas em situação de rua por UF nos anos de 2014-2018 (B)

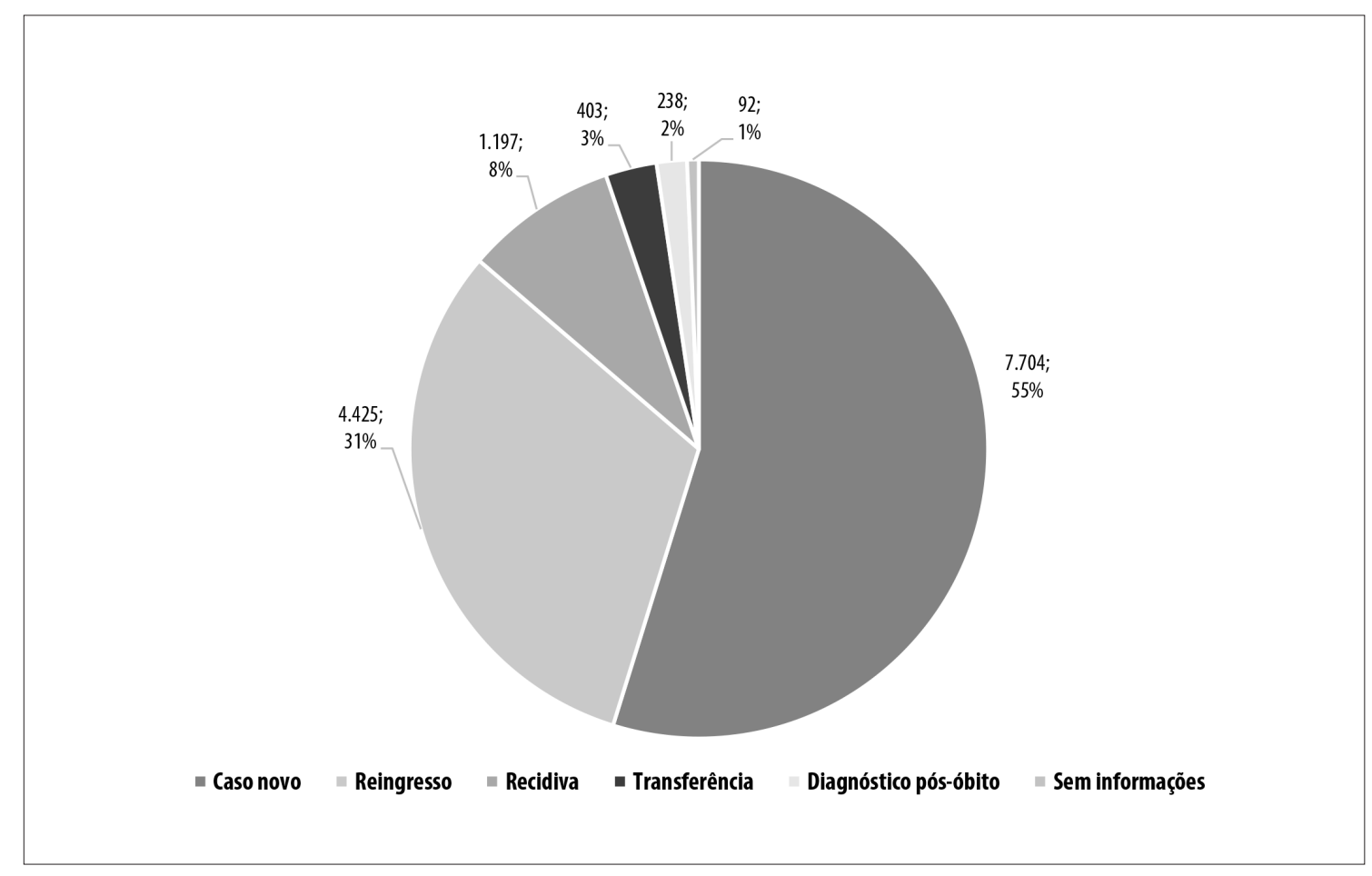

Figura 2 - Número e proporção de casos de tuberculose na população em situação de rua, de acordo com 0 modo de entrada da notificação no Sistema de Informação de Agravos de Notificação (Sinan), Brasil, 2014-2018 
6.355 pessoas, $45,2 \%$ dos casos notificados entre os anos de 2014 e 2018.

Dos casos de tuberculose na PSR registrados no Sinan (Tabela 1), 80,9\% (11.377) foram de pessoas do sexo masculino e $19,1 \%$ (2.682) do sexo feminino. Quanto à faixa etária, 80,7\% (11.347) dos casos ocorreram entre pessoas de 25 a 54 anos, com maior predomínio entre indivíduos na faixa etária dos 35 aos 44 anos $(54,2 \%)$. Considerando-se a raça/cor da pele, predominou a população negra, com 64,1\% (9.003) dos casos, ou seja, 44,2\% (6.209) de pessoas autodeclaradas pardas e 19,9\% (2.794) de pessoas pretas.

Com relação à escolaridade da PSR com tuberculose notificada no Sinan, 46,0\% (6.469) chegaram ao ensino fundamental. Quanto a ser beneficiário de programas sociais do governo, observou-se que $46,2 \%$ (6.489) não recebiam esses benefícios, senão apenas 4,0\% (565); esta variável, entretanto, apresentou 49,8\% (7.005) de PSR sem essa informação (Tabela 1).

Conforme a Tabela 2, a PSR do Brasil registrada no CadÚnico constituía-se de 86,8\% (110.732) de pessoas do sexo masculino e 74,8\% (95.394) de indivíduos concentrados na faixa etária dos 25 aos 54 anos. Sobre a variável 'raça/cor da pele', os dados do CadÚnico apresentaram 51,3\% (65.454) autodeclarados pardos e $16,2 \%$ (20.652) pretos, o que perfez $67,5 \%$ (86.106) de pessoas negras.

A escolaridade, determinada pelo grau de instrução, revelou $62,1 \%(79.258)$ a ter alcaçado o ensino fundamental: 48,4\% (61.779) com ensino fundamental incompleto e 13,7\% (17.479) com ensino fundamental completo. Quanto à renda familiar per capita, 90,2\% (115.073) da PSR encontravam-se em situação de extrema pobreza. Observou-se que 5,7\% (7.167) dessas pessoas apresentavam renda acima de meio salário mínimo e 75,5\% (96.337) eram beneficiárias do Programa Bolsa Família (Tabela 2).

A Figura 3 apresenta os percentuais de desfecho do tratamento dos casos de tuberculose notificados no Sinan entre a PSR no país. No período de 2014 a 2018, mesmo com a redução do abandono do tratamento estimada em 5,5 pontos percentuais, a proporção de indivíduos que alcançaram a cura declinou $5,1 \%$, passando de 39,6\% em 2014 para 34,5\% em 2018. Houve um aumento nos percentuais de desfechos 'ignorado/ em branco' e 'transferência'. Os óbitos por tuberculose também apresentaram elevação, ao longo dos anos estudados, oscilando de 5,8 a 7,5 pontos percentuais
- precisamente o último ano do período analisado, 2018, foi o responsável pelo maior percentual de óbitos encontrado, 7,5\% do total dos casos de tuberculose na população em situação de rua no Brasil.

\section{Discussão}

Este é o primeiro estudo realizado no Brasil, de abrangência nacional, com dados sociodemográficos e sobre a tuberculose na PSR, disponíveis em sistemas de informações. A partir da análise de toda a PSR cadastrada, os resultados revelaram a concentração da PSR e dos casos de tuberculose, no contingente e objeto do estudo, nos estados das regiões Sudeste e Sul do país, com destaque para o Rio Grande do Sul.

0 perfil sociodemográfico dos casos de tuberculose na PSR, com predominância do sexo masculino e da raça/cor da pele negra (pardos e pretos), é similar ao perfil da PSR registrada no CadÚnico. Além disso, foram identificados altos percentuais de reingresso e recidiva após abandono do tratamento e de piores desfechos, a exemplo do aumento nas proporções de óbitos por tuberculose, no período observado.

A saúde da PSR corresponde a uma das interfaces mais críticas da vulnerabilidade na situação de rua, em um contexto de precárias condições socioeconômicas, dificuldade de acesso aos serviços de saúde, maior risco de infecção, adoecimento e piores desfechos clínicos. Elementos históricos, políticos e socioculturais alicerçam relações antagônicas na sociedade brasileira, cada vez mais responsáveis pela desregulação social do capital, esvaziamento dos direitos sociais e crescimento das iniquidades em saúde. ${ }^{17}$ As ações e políticas públicas ofertadas à PSR não deixam de ser influenciadas por pensamentos preconceituosos sobre pessoas em situação de rua, cuja fragilidade não é reconhecida ou devidamente compreendida pela sociedade geral, norteada pela ideologia dominante de que "viver na rua é uma escolha individual". ${ }^{18}$

Os estados do Sudeste e Sul do Brasil apresentaram as maiores concentrações de PSR exposta a precárias condições de vida e comorbidades. Um exemplo dessas condições está na infecção pelo HIV/aids, que aumenta 0 risco da infecção e adoecimento pelo Mycobacterium tuberculosis. Segundo estudo realizado em Porto Alegre, RS, o percentual da PSR acometida pela tuberculose foi quase a metade do total 
Tabela 1 - Perfil sociodemográfico dos casos de tuberculose (TB) em pessoas em situação de rua, Brasil, 2014-2018

\begin{tabular}{|c|c|c|}
\hline \multirow{2}{*}{ Variáveis } & \multicolumn{2}{|c|}{ Pessoas em situação de rua com TB $(n=14.059)$} \\
\hline & $\mathbf{n}$ & $\%$ \\
\hline \multicolumn{3}{|l|}{ Sexo } \\
\hline Masculino & 11.377 & 80,9 \\
\hline Feminino & 2.682 & 19,1 \\
\hline \multicolumn{3}{|l|}{ Faixa etária (em anos) } \\
\hline$\leq 14$ & 67 & 0,5 \\
\hline $15-24$ & 985 & 7,0 \\
\hline $25-34$ & 3.719 & 26,5 \\
\hline $35-44$ & 4.716 & 33,5 \\
\hline $45-54$ & 2.912 & 20,7 \\
\hline $55-64$ & 1.288 & 9,2 \\
\hline$\geq 65$ & 359 & 2,6 \\
\hline \multicolumn{3}{|l|}{ Raça/cor da pele } \\
\hline Branca & 3.733 & 26,5 \\
\hline Preta & 2.794 & 19,9 \\
\hline Amarela & 84 & 0,6 \\
\hline Parda & 6.209 & 44,2 \\
\hline Indígena & 62 & 0,4 \\
\hline Sem informação & 1.177 & 8,4 \\
\hline \multicolumn{3}{|l|}{ Escolaridade } \\
\hline Analfabeto & 715 & 5,1 \\
\hline Ensino fundamental incompleto & 5.881 & 41,8 \\
\hline Ensino fundamental completo & 588 & 4,2 \\
\hline Ensino médio incompleto & 1.268 & 9,0 \\
\hline Ensino médio completo & 374 & 2,7 \\
\hline Ensino superior incompleto e mais & 222 & 1,6 \\
\hline Sem informação & 5.011 & 35,6 \\
\hline \multicolumn{3}{|l|}{ Beneficiário do governo } \\
\hline Sim & 565 & 4,0 \\
\hline Não & 6.489 & 46,2 \\
\hline Sem informação & 7.005 & 49,8 \\
\hline
\end{tabular}


Tabela 2 - Perfil sociodemográfico das pessoas em situação de rua inscritas no Cadastro Único para Programas Sociais (CadÚnico), Brasil, maio de 2019

\begin{tabular}{|c|c|c|}
\hline \multirow{2}{*}{ Variáveis } & \multicolumn{2}{|c|}{ Pessoas em situação de rua $(n=127.536)$} \\
\hline & $\mathbf{n}$ & $\%$ \\
\hline \multicolumn{3}{|l|}{ Sexo } \\
\hline Masculino & 110.732 & 86,8 \\
\hline Feminino & 16.804 & 13,2 \\
\hline \multicolumn{3}{|l|}{ Faixa etária (em anos) } \\
\hline$\leq 15$ & 3.347 & 2,6 \\
\hline $16-24$ & 7.081 & 5,5 \\
\hline $25-44$ & 66.570 & 52,2 \\
\hline $45-54$ & 28.824 & 22,6 \\
\hline $55-64$ & 16.921 & 13,3 \\
\hline$\geq 65$ & 4.793 & 3,8 \\
\hline \multicolumn{3}{|l|}{ Raça/cor da pele } \\
\hline Branca & 40.457 & 31,7 \\
\hline Preta & 20.652 & 16,2 \\
\hline Amarela & 576 & 0,5 \\
\hline Parda & 65.454 & 51,3 \\
\hline Indígena & 296 & 0,2 \\
\hline Sem informação & 101 & 0,1 \\
\hline \multicolumn{3}{|l|}{ Escolaridade } \\
\hline Analfabeto & 13.859 & 10,9 \\
\hline Ensino fundamental incompleto & 61.779 & 48,4 \\
\hline Ensino fundamental completo & 17.479 & 13,7 \\
\hline Ensino médio incompleto & 11.354 & 8,9 \\
\hline Ensino médio completo & 19.781 & 15,5 \\
\hline Ensino superior incompleto e mais & 1.905 & 1,5 \\
\hline Sem informação & 1.379 & 1,1 \\
\hline \multicolumn{3}{|l|}{ Faixa da renda familiar per capita } \\
\hline Extrema pobreza & 115.073 & 90,2 \\
\hline Pobreza & 2.332 & 1,8 \\
\hline Baixa renda & 2.964 & 2,3 \\
\hline Acima de $1 / 2$ salário mínimo & 7.167 & 5,7 \\
\hline \multicolumn{3}{|c|}{ Beneficiário do Programa Bolsa Família } \\
\hline Sim & 565 & 4,0 \\
\hline Não & 6.489 & 46,2 \\
\hline Sem informação & 7.005 & 49,8 \\
\hline
\end{tabular}




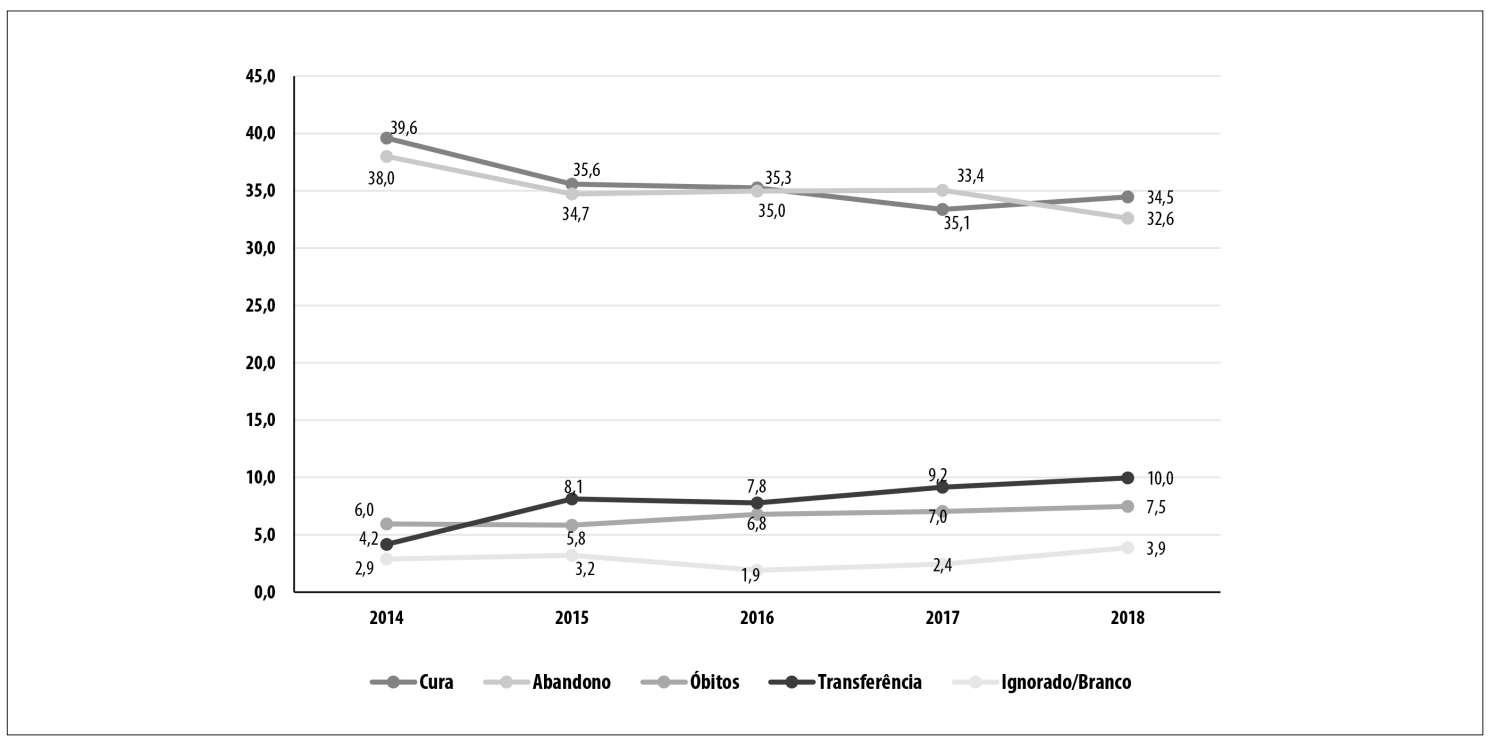

Figura 3 - Desfechos do tratamento dos casos novos de tuberculose na população em situação de rua, Brasil, 2014-2018

de casos na área central daquela capital, no período de 2007 a $2011 .^{19}$

Outro estudo, realizado no estado de São Paulo, identificou associação entre falta de moradia e redução da sobrevida de pessoas com tuberculose, com destaque para a faixa etária economicamente ativa (20 a 40 anos), agravando ainda mais sua condição de vulnerabilidade. ${ }^{20} \mathrm{Em}$ um cenário de aumento do número de pessoas sobrevivendo nas ruas, em razão de diversos fatores, como desemprego e migrações, principalmente nas grandes cidades, é importante considerar se as menores proporções de PSR nos demais estados resultam das lacunas na vigilância dos casos de tuberculose nessa população ou se, de fato, traduzem a realidade do país.

A análise dos dados do Sinan evidencia altos percentuais de recidiva e reingresso no tratamento da tuberculose na PSR. Na percepção de profissionais de saúde da cidade de São Paulo, existem dificuldades na adesão à terapia medicamentosa de longa duração e no monitoramento da saúde dos indivíduos infectados pelo M. tuberculosis, decorrentes da ausência de infraestrutura, baixa escolaridade, alimentação inadequada, desemprego etc. ${ }^{20}$

Estudos, também realizados no estado de São Paulo, revelam um percentual de abandono do tratamento da tuberculose na PSR de 40,5\% no ano de
2014, e aproximadamente 56\% com desfechos desfavoráveis. ${ }^{21}$ No presente trabalho, 0 alto índice de abandono reflete-se no alto percentual de reingresso $\mathrm{e}$ recidiva do tratamento.

Os casos de tuberculose no período analisado ocorreram, majoritariamente, em indivíduos do sexo masculino, negros (pretos e pardos), de baixa escolaridade e em idade economicamente ativa. Na análise das informações da PSR disponíveis no CadÚnico, observou-se um perfil sociodemográfico semelhante ao da PSR com tuberculose notificada no Sinan e, ademais, uma faixa de renda familiar per capita caracterizada como de extrema pobreza. ${ }^{16}$ Corroborando o perfil identificado neste estudo, o censo da PSR de 2008 revelou maior frequência de pessoas do sexo masculino, com idade entre 18 e 54 anos, e predominância da raça/cor da pele negra. 0 censo em questão também revelou uma PSR composta por trabalhadores e uma minoria a depender, como principal fonte de renda, do pedido de dinheiro nas ruas, sem acesso a programas de oferta de renda pelo governo, analfabetos funcionais e exercendo alguma atividade remunerada. ${ }^{4}$

A análise dos fatores sociodemográficos na PSR com tuberculose visibiliza a raça/cor da pele dessas pessoas, em sua maioria negras. Outros estudos realizados no Brasil ${ }^{5,22}$ demonstram a relação entre raça/cor da pele e exclusão social. Considerando-se as diversas 
vulnerabilidades a que a sociedade capitalista expõe as classes menos favorecidas, a PSR, especialmente, apresenta dramas e demandas comumente invisibilizadas e naturalizadas, corroborando situações já sofridas pela população negra ao longo da história da sociedade brasileira. ${ }^{23}$

Em relação ao Programa Bolsa Família, os dados do CadÚnico apontam que um terço dessa população é sua beneficiária. No estudo realizado em Salvador, sobre 0 recebimento de benefícios sociais por pessoas com tuberculose, demonstrou-se que os indivíduos beneficiários de programas sociais tiveram $14,5 \%$ maior sucesso no tratamento da infecção. ${ }^{24}$ Esta análise, quando atrelada às vulnerabilidades descritas aqui, pode indicar caminhos para a melhoria da adesão ao tratamento.

Considerando-se todas as superações necessárias à realização do diagnóstico e tratamento da tuberculose na PSR, os resultados deste estudo evidenciam elevados percentuais de abandono do tratamento e óbitos. Percentuais tão altos reafirmam as conclusões de estudos realizados no Brasil e em países europeus, sobre a condição de viver em situação de rua como agravante para a falência do tratamento da tuberculose e aumento da ocorrência de óbitos. ${ }^{20,25-27}$

0 estudo apresenta limitações, entre elas a impossibilidade de calcular a incidência da tuberculose na PSR: não há estimativas populacionais fidedignas dessa população por UF. Além disso, é preciso ter em conta 0 sub-registro das variáveis 'escolaridade' e 'benefício do governo' na ficha do Sinan, enquanto, no Cadúnico, o registro do recebimento de benefício do governo é obrigatório.

Como pontos fortes da pesquisa, está a possibilidade da comparação de dados de toda a PSR registrada no CadÚnico, em nível nacional, assim como dos dados das notificações de tuberculose das pessoas em situação de rua nos últimos cinco anos. 0 estudo também revela os altos percentuais de desfechos negativos (abandono e óbito) e os casos reincidentes nessa população.

Há uma carência de evidências que permitam aprofundar a discussão sobre a relação dos fatores sociodemográficos e econômicos com o diagnóstico, evolução clínica e desfecho do tratamento da tuberculose na PSR, pelo que se fazem necessárias mais pesquisas, qualitativas e quantitativas, para fundamentar uma ampla discussão, maior visibilidade do problema e decisão segura dos gestores quanto às ações de saúde voltadas a essa população.
Cumpre a estes autores recomendar o fortalecimento das redes socioassistenciais e de saúde, em parceria com as representações sociais instituídas, para 0 atendimento à PSR. Extremamente necessário e importante é o desenvolvimento de ações de reparação social que possibilitem 0 acesso a alimentação e moradia dignas, e promoção da cidadania.

No âmbito do Sistema Único de Saúde, é fundamental eliminar as barreiras existentes entre a PSR e os serviços prestados pelo sistema, mediante o fortalecimento da Atenção Básica como ação potencializadora na identificação precoce da infecção e tratamento monitorado, tendo como uma de suas estratégias 0 tratamento diretamente observado. ${ }^{13,28}$

Finalmente, é necessário qualificar os dados sobre a PSR disponíveis nos sistemas de informações, favorecendo a tomada de decisão sobre políticas públicas para redução da invisibilidade dessa população no Brasil. A sensibilização das equipes de saúde que realizam o diagnóstico e o preenchimento dos formulários é tão imprescindível para a qualificação da informação quanto para a o desenho de políticas intersetoriais efetivas, na organização de uma rede de atenção integral à saúde da PSR.

0s resultados do estudo permitem concluir que a tuberculose permanece como um grave problema de Saúde Pública, que agrava a condição de sobrevivência nas ruas, funcionando como um marcador da violação do acesso e direito à saúde da população em situação de rua no Brasil.

\section{Agradecimentos}

A Felipe Nery, pela elaboração dos mapas deste estudo.

\section{Contribuição dos autores}

Almeida MVG e Santos SD contribuíram na concepção e delineamento do problema e desenho metodológico do estudo. Silva TO e Vianna PJS contribuíram no levantamento, estruturação dos dados e redação da primeira versão do manuscrito. Nery JS realizou a revisão geral da primeira versão e contribuiu na análise dos dados. Todos os autores aprovaram a versão final e são responsáveis por todos os aspectos do estudo, incluindo a garantia de sua fidedignidade e integridade. 


\section{Referências}

1. Brasil. Política Nacional para Inclusão da Pessoa em Situação de Rua [Internet]. Brasília: Ministério do Planejamento, Orçamento e Gestão. Secretaria de Direitos Humanos; 2008. [citado 2019 fev 5]. 25 p. Disponível em: <http://www.mpsp.mp.br/portal/page/ portal/cao_civel/acoes_afirmativas/inclusaooutros/ aa_diversos/Pol.Nacional-Morad.Rua.pdf>.

2. Brasil. Ministério da Saúde (BR). Secretaria de Gestão Estratégica e Participativa. Departamento de Apoio à Gestão Participativa. Saúde da população em situação de rua: um direito humano. Brasília: Ministério da Saúde; 2014.

3. Brasil. Ministério da Saúde (BR). Secretaria de Vigilância em Saúde. Departamento de Vigilância Epidemiológica. Boletim epidemiológico sobre população em situação de rua e violência: uma análise das notificações do Brasil de 2015 a 2017. Brasília: Ministério da Saúde; 2019 jun (50).

4. Brasil. Ministério do Desenvolvimento Social e Combate à Fome (BR). Rua: aprendendo a contar. Pesquisa Nacional sobre a População em Situação de Rua. Brasília: Ministério do Desenvolvimento Social e Combate à Fome; 2009

5. Natalino, MAC. Estimativa da população em situação de rua no Brasil (setembro de 2012 a março de 2020) [Nota Técnica]. Brasília: Instituto de Pesquisa Econômica Aplicada; 2020. 20 p.

6. Brasil. Casa Civil. Decreto Federal $n^{0} 7053$, de 23 de dezembro de 2009. Institui a Política Nacional para a População em Situação de Rua e seu Comitê Intersetorial de Acompanhamento e Monitoramento, e dá outras providências. Diário Oficial da República Federativa do Brasil, Brasília (DF), 2009 dez 24; p. 16.

7. Barbosa, J. C. Implementação das políticas públicas voltadas para a população em situação de rua: desafios e aprendizados. Programa de Pós-Graduação em Políticas Públicas e Desenvolvimento, Instituto de Pesquisa Econômica Aplicada. Brasília, 2018.

8. Brasil. Ministério da Saúde (BR). Plano operativo para implementação de ações em saúde da população em situação de rua 20122015. Brasília: Ministério da Saúde; 2012.

9. Brasil. Ministério da Saúde. Portaria n ${ }^{0}$ 2436, de 21 de setembro de 2017. Aprova a Política Nacional de Atenção Básica, estabelecendo a revisão de diretrizes para a organização da Atenção Básica, no âmbito do Sistema Único de Saúde (SUS). Diário Oficial da República Federativa do Brasil, Brasília (DF), 2017.

10. World Health Organization (WHO). Global tuberculosis report. New York: WHO; 2020. Disponível em: < https://www.who.int/publications/i/item/ who-consolidated-guidelines-on-tuberculosis-module1-prevention-tuberculosis-preventive-treatment $>$. Acesso em: 25 de agosto de 2020.

11. Salvador. Prefeitura Municipal. Relatório da pesquisa sobre a população em situação de rua no município de Salvador-Ba/ Prefeitura Municipal de Salvador. 1. ed. - Salvador: Programa Salvador Cidadania, 2010. 93p.

12. Ranzani OT, Carvalho CR, Waldman EA, Rodrigues LC. The impact of being homeless on the unsuccessful outcome of treatment of pulmonary TB in São Paulo State, Brazil. BMC Med. 2016 mar 23; 14:41.

13. Brasil. Ministério da Saúde (BR). Secretaria de Vigilância em Saúde. Departamento de Vigilância das Doenças Transmissíveis. Manual de Recomendações para o Controle da Tuberculose no Brasil. Brasília: Ministério da Saúde; 2018.

14. Brasil. Ministério da Cidadania (BR). Relatórios de Informações Sociais. Consulta, Seleção e Extração de informações do Cadastro Único - CECAD. Brasília: Ministério da Cidadania; 2020. Disponível em: https://aplicacoes.mds.gov.br/sagi/cecad20/ tab_cad.php. Acesso em: 02 de maio de 2019.

15. Brasil. Ministério da Saúde (BR). Informações de saúde (TABNET) [Internet]. Brasília: Ministério da Saúde; 2016. [citado em 2016 set 15]. Disponível em: <http:// tabnet.datasus.gov.br/cgi/tabcgi.exe?sinannet/cnv/ tubercbr.de >/. Acesso em 20 de setembro de 2020.

16. Brasil. Ministério da Cidadania (BR). Guia de Cadastramento de Pessoas em Situação de Rua. Brasília: Ministério da Cidadania; 2020. Disponível em: https://www.mds.gov.br/ webarquivos/arquivo/cadastro_unico/_Guia_ Cadastramento_de_Pessoas_em_Situacao_de_Rua. pdf. Acesso em: 02 de setembro de 2020.

17. Geolin, George Francisco. Crise do capital, precarização do trabalho e impactos no Serviço Social. Serv. Soc. Soc., São Paulo, n. 118, p. 239-264, Jun 2014.

18. Serafino I, Luz LCX. Políticas para a população adulta em situação de rua: questões para debate. Revista Katálysis, v. 18, n. 1, p. 74-85, 2015. 
19. Peruhype, RC et al. Distribuição da tuberculose em Porto Alegre: análise da magnitude e coinfecção tuberculose-HIV. Revista da Escola de Enfermagem da USP, v. 48, n. 6, p. 1035-1043, 2014.

20. Ranzani OT, Rodrigues LC, Bombarda S, Minto CM, Waldman EA, Carvalho CRR. Long-term survival and cause-specific mortality of patients newly diagnosed with tuberculosis in São Paulo state, Brazil, 2010-15: a population-based, longitudinal study. Lancet Infect Dis. 2020;20(1):123-132. doi:10.1016/S1473-3099(19)30518-3

21. São Paulo (Cidade). Estratégias no enfrentamento da tuberculose na população em situação de rua na cidade de São Paulo. In: Assembleia da Rede de Comitês Tb, 7. 2015, Brasília. Anais: Brasília, 2015. Disponível em: https://drive.google.com/ file/d/0B0CE2wqdEaR-Wm1TLTVhQ29FWWc/ view. Acesso em: 26 de setembro de 2020 .

22. Mattos RM, Ferreira RF. Quem vocês pensam que (elas) são? Representações sobre as pessoas em situação de rua. Psicol Soc. 2004;16(2):47- 58.

23. Silva, TD; Natalino, M; Pinheiro, MB. População em situação de rua em tempos de pandemia: um levantamento de medidas municipais emergenciais [Nota Técnica]. Brasília: Instituto de Pesquisa Econômica Aplicada; 2020. 26 p.
24. Andrade KVF, Nery JS, Araújo GS, Barreto ML, Pereira SM. Associação entre desfecho do tratamento, características sociodemográficas e benefícios sociais recebidos por indivíduos com tuberculose em Salvador, Bahia, 2014-2016. Epidemiol. Serv. Saúde. 2019; 28(2): e2018220.

25. Nordentoft M, Wandall-Holm N. 10 year follow up study of mortality among users of hostels for homeless people in Copenhagen. BMJ 2003; 327: 81-83. Disponível em: https:/www.ncbi.nlm.nih. gov/pmc/articles/PMC164916/pdf/el-gp81.pdf.

26. Beijer U, Wolf A, Fazel S. Prevalence of tuberculosis, hepatitis $\mathrm{C}$ virus, and HIV in homeless people: a systematic review and meta-analysis. Lancet Infect Dis 2012;12: 859-70. doi:1473-3099(12)70177-9

27. Bernard C, Sougakoff W, Fournier A, Larnaudie S, Antoun F, Robert J, Brossier F, Truffot-Pernot C, Jarlier $\mathrm{V}$, Veziris N. Impact of a 14-year screening programme on tuberculosis transmission among the homeless in Paris. Int J Turerc Lung Dis, 2012. 16(5):649-655.

28. Alecrim TFA, Mitano F, Reis AA, Roos CM, Palha PF, Protti-Zanatta ST. Experiência dos profissionais de saúde no cuidado da pessoa com tuberculose em situação de rua. Rev. esc. enferm. USP. 2016; Out 50(5): 808-815. 


\section{Abstract}

Objective: To characterize the sociodemographic profile of street people (SP) registered on the Single Register for Social Programs (CadÚnico) as at 2019 and tuberculosis morbidity in this population notified on the Notifiable Health Conditions Information System (Sinan) from 2014 to 2018. Methods: This was a descriptive study carried out with data from Sinan and CadÚnico. Results: 127,536 people registered on CadÚnico and 14,059 with tuberculosis notified on Sinan were included in the study. Higher concentrations of SP were found in the states of the South and Southeast regions. In the two subpopulations analyzed, there was a predominance of male SP (CadÚnico = 86.8\%; Sinan = 80.9\%), people of Black race/skin color (CadÚnico $=67.5 \%$; Sinan $=$ 64.1\%) and with incomplete high school education or less (CadÚnico $=81.9 \%$; Sinan $=60.1 \%$ ). The bigh treatment dropout proportion (39.0\%) was reflected in the high percentage of reentry and relapse. Conclusion: Tuberculosis continues to be a serious Public Health problem that aggravates survival conditions on the streets of Brazil.

Keywords: Homeless Persons; Social Vulnerability; Epidemiology, Descriptive; Tuberculosis.

\section{Resumen}

objetivo: Caracterizar el perfil sociodemográfico de la población sin hogar (PSR) inscrita en el Registro Único para Programas Sociaes (CadÚnico) basta 2019 y la morbilidad tuberculosa en esta población notificada en el Sistema de Información de Agravamientos de Notificación (Sinan) de 2014 a 2018. Métodos: Estudio descriptivo realizado con datos del Sinan y CadÚnico. Resultados: Se incluyeron 127.536 personas registradas en el CadÚnico y 14.059 con tuberculosis notificada en el Sinan. Se observaron concentraciones más altas de PSR en los estados del Sur y Sudeste. En las dos subpoblaciones analizadas, bubo predominio de PSR masculina (CadÚnico = 86,8\%; Sinan = 80,9\%), raza) color negra (Cadúnico $=67,5 \%$; Sinan $=64,1 \%$ ) y baja escolaridad, hasta la secundaria incompleta (Cadúnico $=81,9 \%$; Sinan $=60,1 \%)$. Se evidenció una alta proporción de abandono (39,0\%) del tratamiento por el alto porcentaje de reingreso y recaída. Conclusión: La tuberculosis sigue siendo un problema grave de Salud Pública, que agrava la condición de supervivencia en las calles de Brasil.

Palabras clave: Personas sin Hogar; Vulnerabilidad Social; Epidemiología, Descriptiva; Tuberculosis.

Recebido em 25/07/2020

Aprovado em 17/11/2020 